\title{
Rehabilitación cardiaca en época COVID-19. Programas. Resultados
}

\author{
Cardiac rehabilitation in the COVID-19 time. Programs. Results \\ José Maroto-Montero ${ }^{*}$, Mercedes Coello-Cremades ${ }^{1}$, Laura Martín-Argomanid', Marta Maroto-de-Pablo', \\ Miguel Gómez-Martínez ${ }^{1}$, Concepción Paredes-Camargo ${ }^{1}$ y Carmen de-Pablo-Zarzosa² \\ ${ }^{1}$ Unidad de Rehabilitación Cardiaca, Instituto de Rehabilitación Funcional La Salle; ${ }^{2}$ Unidad de Rehabilitación Cardiaca, Servicio de Cardiología, \\ Hospital Ramón y Cajal. Madrid, España
}

\begin{abstract}
Resumen
Introducción: La pandemia de enfermedad por coronavirus 2019 (COVID-19) ha incidido de forma negativa en los programas de rehabilitación cardiaca (PRC) españoles. Objetivo: Este trabajo tiene como objetivo fundamental el analizar la posibilidad de mantenerlos abiertos y como secundario valorar si se mantienen los beneficios demostrados a nivel físico y psicológico. Métodos: Analizamos los resultados de nuestro PRC en el año 2020 (119 pacientes) y los comparamos con los del año 2019 (121 pacientes), libre de COVID-19. Se comparan distintas variables generales, y los resultados obtenidos en capacidad física, a nivel psicológico, así como las complicaciones y abandonos. Resultados: No existen diferencias significativas entre ambos grupos en la edad (61.6 vs. 61.5), sexo (mujeres: 12.6 vs. 14.8\%), diagnóstico de cardiopatía isquémica (106 vs. 99) y fracción de eyección de ventrículo izquierdo (55.9 vs. 55.8\%). La ansiedad media-alta fue superior al inicio $(p=0.02)$ y final ( $p=0.002$ ) del programa en el año 2019, así como las puntuaciones del patrón de conducta tipo $A$ ( $p=0.041$ vs. 0.034 ). El porcentaje de depresión fue similar. Más del 95\% de los pacientes del año 2020 referían miedo al contagio. La capacidad funcional aumentó, pero menos en el año $2020(p=0.001)$. La duración del programa fue superior en el año 2020 $(p=0.001)$. Los abandonos (15 vs. 11 pacientes) no mostraron diferencias significativas. Conclusiones: Los PRC en época COVID-19 son posibles y siguen mostrando beneficios. La duración del programa fue mayor por periodos de falta de asistencia. La menor capacidad física puede estar relacionada con el uso de la mascarilla.
\end{abstract}

Palabras clave: Rehabilitación cardiaca. Pandemia COVID-19. Abandonos. Miedo al contagio.

\begin{abstract}
Introduction: COVID-19 pandemic has had a negative impact on Spanish Cardiac Rehabilitation Programmes (CRP). Objectives: The main objective of this study is to analyze the possibility of keeping these units open and, secondly, to assess whether demonstrated physical and psychological benefits are preserved. Methods: We analyzed results of our CRP in 2020 (119 patients) and compared them with those of 2019 (121 patients), year free of Covid. We compare different general variables, and results obtained in physical capacity, psychological level, as well as complications and dropouts. Results: There were no significant differences between two groups in age (61.6 vs. 61.5), sex (women: $12.6 \%$ vs. 14.8\%), diagnosis of
\end{abstract}

Correspondencia:

José Maroto-Montero*

E-mail: jmmmcp@yahoo.esjm.maroto@irflasalle.es CC BY-NC-ND (http://creativecommons.org/licenses/by-nc-nd/4.0/).
Disponible en internet: 16-12-2021 Arch Cardiol Mex. 2021;91(Supl):25-33 www.archivoscardiologia.com 
ischemic heart disease (106 vs. 99) and left ventricular ejection fraction (55.9\% vs. 55.8\%). Mean-high anxiety was higher at baseline $(p=0.02)$ and endline $(p=0.002)$ of program in 2019 , as well as type $A$ behavior pattern scores $(p=0.041$ and 0.034). Percentage of depression was similar. More than $95 \%$ of patients in 2020 reported fear of infection. Functional capacity increased, but less in 2020 ( $p=0.001$ ). Programme duration was longer in 2020 ( $p=0.001)$. Dropouts (15 vs. 11 patients) showed no significant differences. Conclusions: CR programmes in COVID-19 era are possible and continue to show benefits. The duration of programme was longer due to periods of non-attendance. Lower physical capacity may be related to mask use.

Keywords: Cardiac rehabilitation. COVID-19 pandemic. Dropouts. Fear of infection.

\section{Introducción}

La pandemia de enfermedad por coronavirus 2019 (COVID-19) ha cambiado la forma de vida en el mundo actual, como consecuencia de la alta incidencia de morbimortalidad directa o indirecta, y por los adversos efectos a nivel socioeconómico.

La sobrecarga de ingresos, como consecuencia de la infección, ha incrementado la ocupación desmedida de las camas de los servicios hospitalarios y de las unidades de cuidados intensivos. Un alto porcentaje de profesionales de distintas especialidades, de los hospitales y centros de salud, han sido movilizados para el tratamiento de los contagiados. Los servicios de cardiología tuvieron que adaptarse a la situación.

La Asociación de Cardiología Intervencionista de la Sociedad Española de Cardiología ha descrito algunos aspectos negativos de la asociación del síndrome coronario agudo (SCA) con las infecciones por el coronavirus $^{1,2}$. Han resaltado que: a) hasta un $10 \%$ de los pacientes con infarto agudo de miocardio (IAM) presentaron COVID-19, durante la primera ola; b) hubo una disminución en el número de pacientes con IAM que acudían a los servicios de urgencia, se cuantificó en un $30 \%$ durante los meses de marzo y abril de 2020 , en comparación con los datos del año 2019, y c) la mortalidad por IAM con elevación del ST (IAMCEST) se duplicó con respecto al mismo periodo de tiempo de 2019.

Las unidades de rehabilitación cardiaca (URC) han tenido que modificar sus actuaciones y en muchos casos cerrar temporalmente, como consecuencia del confinamiento y posteriormente al tener que cumplir los mínimos necesarios aconsejados (espacios, vestuarios, etc.) por las secciones específicas de distintas sociedades de cardiología ${ }^{3,4}$.

Los componentes de la URC, del Instituto de Rehabilitación Funcional La Salle de Madrid, hemos considerado que quizás pueda tener interés el describir las modificaciones realizadas en nuestro programa, durante el año 2020, y comparar los resultados con los obtenidos en el 2019, libre de COVID-19.

El objetivo principal del presente estudio es analizar la posibilidad de mantener abiertas las URC durante la época COVID-19, adecuando las actuaciones a los periodos de confinamiento. El objetivo secundario es estudiar si se mantienen los beneficios demostrados a nivel físico y psicológico, de los programas de rehabilitación cardiaca (PRC).

\section{Material y métodos}

El presente artículo prospectivo (año 2020) y retrospectivo (año 2019) describe las modificaciones efectuadas en nuestro PRC multidisciplinario en época de pandemia, relacionadas con la política sanitaria y la evolución de la enfermedad.

Se han analizado distintos parámetros generales, como: edad, sexo, profesión, diagnósticos, fracción de eyección del ventrículo izquierdo (FEVI), presentación de complicaciones, duración del programa, realización de marchas domiciliarias, el porcentaje de abandonos y las razones de ello.

En los pacientes con SCA: el retraso entre la aparición del dolor y la realización de la revascularización percutánea o quirúrgica, y si estas fueron completas o incompletas.

Se tabulan distintos datos de la prueba de esfuerzo inicial y final (realizada sobre tapiz, según protocolo de Bruce en rampa, y siguiendo las recomendaciones de prevención de infección COVID-19 durante el año 2020): capacidad física medida en MET, la aparición o no de arritmias, la respuesta de la tensión arterial y la presencia de positividad clínica y/o eléctrica.

Se analizó el porcentaje de alteraciones psicológicas valoradas mediante entrevistas individuales y con el pase de cuestionarios psicológicos validados que analizan la ansiedad (Hamilton) ${ }^{5}$, la depresión (Beck) ${ }^{6}$ y el patrón de conducta tipo $\mathrm{A}$ (Bortner) ${ }^{7}$, al inicio y fin del 
programa. De igual modo, se valoró la necesidad o no de tratamiento especializado, siguiendo las normas de trabajo de nuestra subunidad psicológica-psiquiátrica ${ }^{8}$.

En los pacientes del año 2000 se estudia la existencia de infección por coronavirus 2 del síndrome respiratorio agudo grave (SARS-CoV-2) previa y el tiempo transcurrido entre una prueba de reacción en cadena de la polimerasa (PCR) negativa y la entrada en el programa. De forma similar, la infección aparecida durante el periodo de tratamiento, la evolución y el tiempo transcurrido entre la última PCR negativa y el reingreso en el programa. Así mismo, se analiza la existencia o no de contagio al resto de pacientes o a los profesionales. En todos los pacientes se describen los controles del entrenamiento físico a nivel objetivo (frecuencia cardiaca de entrenamiento [FCE]) y subjetivo con la escala de Borg 9 .

Los enfermos acuden remitidos por sus cardiólogos. La mayor parte pertenecen a sociedades médicas y muy pocos son privados. En España se consideran privados los que abonan directamente las actuaciones médicas; el coste es superior al abonado por las Sociedades. El contrato habitual con las sociedades tiene una duración de 8-12 semanas, e incluye tres sesiones/ semana de entrenamiento, ocho charlas-coloquio y ocho sesiones de aprendizaje de relajación. La duración del programa aumenta en caso de necesidad, sin incidir en los costos de este.

En el presente estudio se cuantifica como duración del programa, las semanas transcurridas entre el inicio y alta del programa (una vez cumplidas las sesiones contratadas).

Desde la creación de las distintas unidades desarrollada por nuestro grupo, la primera en noviembre de 1979 , los pacientes firman el consentimiento informado, acreditado en la actualidad por el IRF La Salle, dependiente de la Universidad Autónoma de Madrid.

La pandemia nos ha obligado a modificar nuestro PRC en tres ocasiones durante el año 2020:

- EI PRC multidisciplinario clásico de nuestra unidad, basado en publicaciones previas ${ }^{10}$, se realizó durante el año 2019 y los dos primeros meses del 2020. Consiste en: a) entrenamiento físico supervisado en el centro, tres días en semana, e individualizado (marchas diarias domiciliarias); b) actuación psicológica con técnicas de relajación, y c) programa educativo sobre modificación del estilo de vida y control de factores de riesgo coronario.

El cierre de las instalaciones durante el periodo de confinamiento (13 de marzo al 20 de junio) nos motivó a desarrollar un tratamiento por teleasistencia.

- La telerrehabilitación se inició el día 23 de marzo y se mantuvo hasta el 19 de junio. Se incluyó a los pacientes que estaban realizando el protocolo previo, así como a enfermos que solicitaron, por teléfono, iniciar la rehabilitación. Consistió en:

- Entrenamiento físico (lunes y jueves): tabla de ejercicio de 30 minutos de duración seguida de paseos por la casa de forma continuada o intermitente, durante 30-40 minutos. Algunos pacientes tenían bicicleta estática o cintas en su domicilio. En todos ellos, los niveles subjetivos de esfuerzo (escala de Börg) se tomaron muy en cuenta; también fue utilizada en los nuevos enfermos, tras explicársela por teleasistencia. Se aconsejó mantener este entrenamiento el resto de los días de la semana.

- Charlas coloquio de una hora de duración (martes).

- Pautas de relajación durante 30 minutos (miércoles). Una vez aprendidas se les aconsejó que las realizaran por la mañana y la noche todos los días.

- La reapertura de las instalaciones dio lugar al desarrollo de un nuevo programa.

- PRC de reapertura: se inició el día 22 de junio y se mantiene hasta la actualidad. Con base en lo aconsejado por las secciones de rehabilitación cardiaca de las sociedades española y francesa de cardiología ${ }^{3,4}$, los enfermos se dividieron en ocho grupos a la hora de realizar el entrenamiento físico, para poder mantener un espacio individual de $2 \mathrm{x}$ 2 metros en la tabla de fisioterapia, y una distancia de 2 metros durante el ejercicio en aparatos (cicloergómetro y cinta) (Fig. 1).

- En función de espacio del gimnasio, el número máximo de pacientes, por grupo, es de ocho. Acuden a realizar el entrenamiento en sesiones de 60 minutos, los lunes y miércoles o los martes y jueves a las 10, 11, $12013 \mathrm{~h}$.

- Los enfermos fueron informados por la secretaria del grupo que les correspondía. Así mismo, cumplimentaron un cuestionario de detección de infección por SARS-CoV- $2^{4}$. En la actualidad, los pacientes nuevos responden a este cuestionario por teléfono, cuando solicitan cita para realizar el programa presencial.

- En la primera entrevista, la secretaria les pasa el cuestionario de beneficios-riesgos ${ }^{4}$. Posteriormente, son revisados en consulta por el cardiólogo. La enfermera finaliza las actuaciones realizando una historia de enfermería, complementada con información sobre el programa, asignación de un grupo, dietas y entrega de cuestionarios psicológicos.

\section{Normas en el gimnasio}

Durante las sesiones de entrenamiento, el gimnasio se ventila cada diez minutos, abriendo la puerta que 


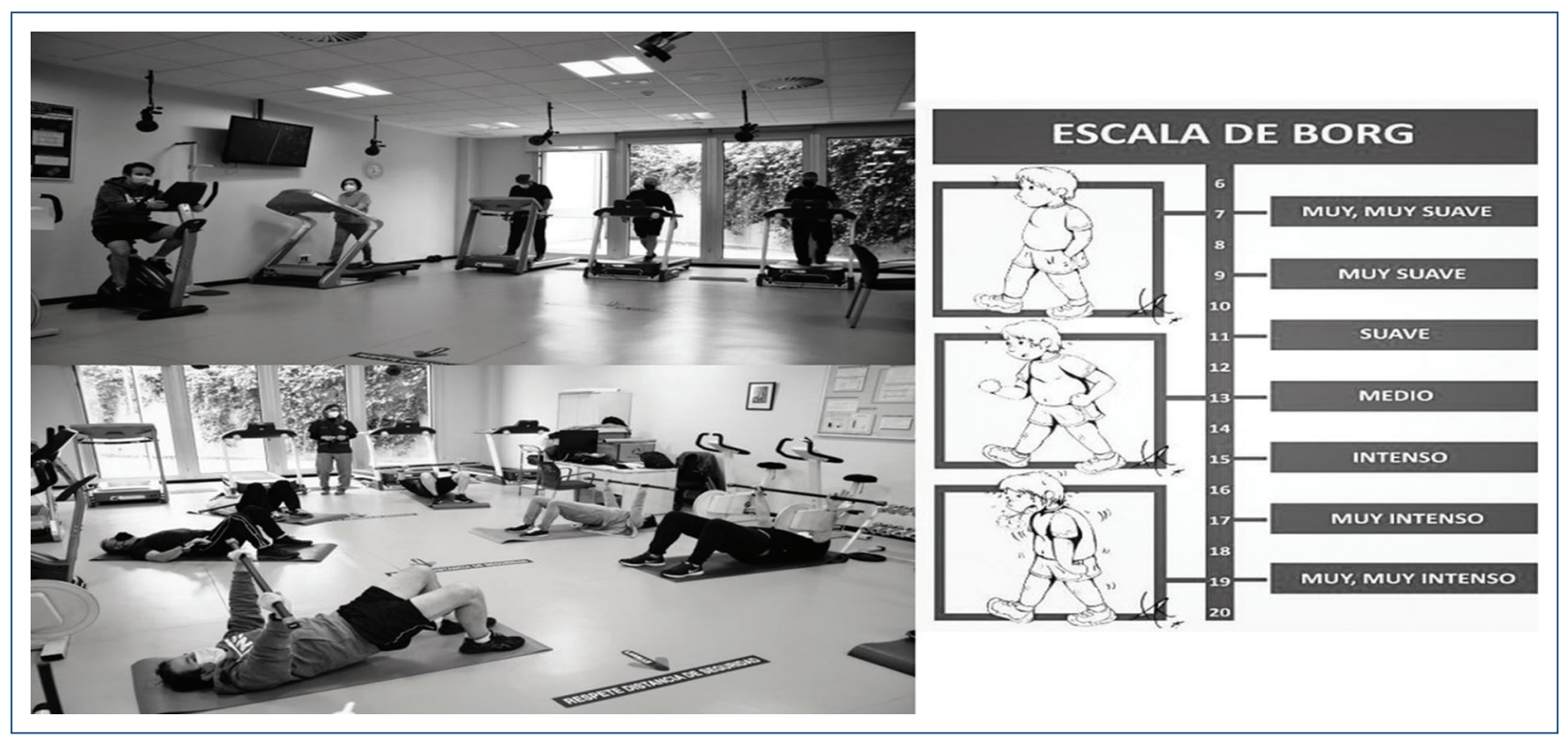

Figura 1. Sesión de entrenamiento y niveles a los que se efectúa el ejercicio. La frecuencia cardiaca, en los aparatos (cinta y ergómetro) se mantendrá alrededor del 75-85\% de la alcanzada en la prueba de esfuerzo. La sensación subjetiva de esfuerzo es fundamental, en rehabilitación cardiaca, sobre todo en época COVID-19. Aconsejamos unas puntuaciones de 12-14 de la escala de Börg. COVID-19: enfermedad por coronavirus 2019.

conecta el gimnasio con un amplio patio exterior. En la época de invierno la calefacción permanece encendida y en verano, el aire acondicionado.

Los pacientes, en la entrevista inicial con la enfermera, reciben consejos y normas para disminuir la posibilidad de contagio. Es necesario comunicar a la URC, llamando por teléfono, si aparecen algunas de las anomalías expresadas en el cuestionario inicial. Antes de iniciar las sesiones de entrenamiento se cuantifica la temperatura y se pregunta sobre la existencia sintomatología clínica cardiológica o infecciosa.

Los profesionales utilizan mascarilla quirúrgica permanente, máscara y visor o gafas durante las actividades colectivas. Los ergómetros no están orientados hacia el personal y se analizan los flujos de aire causados por el aire acondicionado, la calefacción y la apertura de puertas del gimnasio.

\section{Pruebas de esfuerzo y sesiones de entrenamiento}

El paciente recibe las informaciones previas habituales, complementadas con otras específicas de la época COVID-19, como son: a) llevar la mascarilla quirúrgica durante la estancia en las instalaciones, el entrenamiento y las pruebas de esfuerzo; b) lavarse las manos y limpiar las esterillas antes de entrenar en el suelo, así como las zonas de sujeción en bicicleta y banda, con solución hidroalcohólica; c) si tiene que hablar o preguntar, al médico o la enfermera, debe mantener la cabeza mirando hacia adelante, y d) medirse la tensión arterial y la frecuencia cardiaca, al inicio de la sesión, en los tensiómetros de brazo digital existentes en el gimnasio.

No se efectúan ergometrías con consumo de oxígeno, por el riesgo de contagio y dificultades para la limpieza de los sistemas de medición.

El médico y la enfermera deben: a) lavarse las manos con solución hidroalcohólica; b) ponerse guantes de nitrilo, gafas, mascarilla FFP2, gorro para el pelo, bata de papel médico e impermeable para la enfermería y calzas, y c) minimizar las tomas de tensión arterial a estrictamente necesarias. Colocarse en paralelo al paciente mientras esté caminando o corriendo.

El entrenamiento físico supervisado incluye dos sesiones semanales de $1 \mathrm{~h}$ de duración, efectuadas en el gimnasio del centro. En cada sesión se realiza una tabla de fisioterapia y entrenamiento aeróbico en tapiz o bicicleta estática. La FCE se calcula con base en los resultados de las ergometrías realizadas al inicio y al final del programa.

Al finalizar la sesión cada paciente se autoevalúa la sensación subjetiva de esfuerzo en la escala de Borg, en una gráfica individual. Aconsejamos puntuaciones de 12 a 14. El análisis de la Borg es imprescindible en los pacientes que inician el PRC tras haber estado 
infectados por SARS-CoV-2, y en los que reinician el entrenamiento después de pasar la infección (2-3 semanas después de una PCR negativa).

Las mascarillas, y la posibilidad de que la infección haya dejado secuelas pulmonares, aconsejan una vigilancia cercana de la presentación de disnea durante el esfuerzo.

Sesiones de educación terapéutica y de relajación: Los grupos se conectan, por teleconferencia, los viernes de 10 a 12 horas, para charlas-coloquio (90 minutos) y relajación (30 minutos).

\section{Análisis estadísticos}

Todos los análisis estadísticos se llevaron a cabo con el programa IBM SPSS Statistics 26.0. En primer lugar, se estudió la muestra mediante estadística descriptiva, empleando porcentajes para las variables cualitativas, y la media y desviación estándar para las cuantitativas. Se compararon diversas variables de los pacientes que habían realizado el PRC en 2019, con los que lo habían hecho en el 2020 durante la pandemia. Se realizó mediante un análisis bivariante usando el test exacto de Fisher y la prueba de chi cuadrada para las variables cualitativas y la prueba $t$ de Student para las cuantitativas.

Los datos de los pacientes fueron codificados para asegurar anonimizar toda la información almacenada en la base de datos del estudio y la incapacidad de inferirla a la identidad de los participantes.

\section{Resultados}

En el año 2019 ( 1 de enero al 31 de diciembre) se rehabilitó a 121 pacientes. En el 2020 (1 de enero al 31 de diciembre) se incluyeron 119. Las características basales de los pacientes de ambos grupos no mostraron diferencias significativas desde el punto de vista estadístico, a excepción de la duración del programa (Tabla 1).

El porcentaje de pacientes que hacían el programa de marchas domiciliario fue superior en el año 2020 (89\%) al compararlo con el 2019 (78\%), con una $p=0.017$.

Ningún paciente estaba vacunado. La primera vacuna en España se inyectó a una mujer de 96 años, el día 27 de diciembre del año 2020.

\section{Año 2019}

La duración media de los programas fue de 9.36 semanas. En 10 pacientes la estancia fue de 12 a 20,
Tabla 1. Características basales de los pacientes en los años 2019 y $2020^{*}$

\begin{tabular}{|c|c|c|c|}
\hline Variable & $\begin{array}{c}\text { Año } 2019 \\
\text { (121 } \\
\text { pacientes) }\end{array}$ & $\begin{array}{c}\text { Año } 2020 \\
\text { (119 } \\
\text { pacientes) }\end{array}$ & $\mathbf{p}$ \\
\hline Varón, n (\%) & $103(85.1)$ & $104(87.3)$ & \\
\hline Mujer, n (\%) & $18(14.8)$ & $15(12.6)$ & \\
\hline Edad (años), media (DE) & $61.5(9)$ & $61.6(9.3)$ & \\
\hline $\begin{array}{l}\text { Duración programa, } \\
\text { media (DE) }\end{array}$ & $9.3(2.2)$ & $12.1(5.8)$ & 0.001 \\
\hline Abandonos & 11 (9) & $15(12.6)$ & \\
\hline $\begin{array}{l}\text { Profesión } \\
\text { Cuello blanco, n (\%) } \\
\text { Cuello azul, n (\%) } \\
\text { Autónomos, n (\%) }\end{array}$ & $\begin{array}{l}88(72.7) \\
23(19) \\
10(8.2)\end{array}$ & $\begin{array}{c}93(78.1) \\
10(8.4) \\
16(13.4)\end{array}$ & \\
\hline $\begin{array}{l}\text { Diagnóstico } \\
\text { SCACEST, n (\%) } \\
\text { SCASEST, n (\%) } \\
\text { Angina esfuerzo, n (\%) } \\
\text { Cirugía valvular, n (\%) } \\
\text { MCD, n (\%) } \\
\text { Otras, n (\%) }\end{array}$ & $\begin{array}{c}40(33) \\
10(8.2) \\
49(40.4) \\
12(9.9) \\
4(3.3) \\
6(4.9)\end{array}$ & $\begin{array}{c}48(40.3) \\
19(15.9) \\
39(32.7) \\
7(5.8) \\
2(1.6) \\
4(3.3)\end{array}$ & \\
\hline
\end{tabular}

*Las variables cualitativas se expresan en número (porcentaje), las variables cuantitativas en media (desviación estándar). Diferencias estadísticamente significativas $P<0.05$.

DE: desviación estándar; MCD: miocardiopatía dilatada; n: número; SCACEST: síndrome coronario agudo con elevación del ST; SCASEST: síndrome coronario agudo sin elevación del ST.

como consecuencia de baja capacidad funcional secundaria a episodios de insuficiencia cardiaca y patologías añadidas a otros niveles (respiratorio, osteomuscular).

Once pacientes abandonaron el tratamiento: a) uno con disección aórtica, torácica y abdominal, operada, no volvió después de la primera semana; b) cuatro en la tercera o cuarta; c) cuatro entre la quinta y la sexta, $y$ d) dos finalizaron el programa, pero no acudieron a efectuar la prueba de esfuerzo de alta.

Las razones para el abandono fueron: dolores osteomusculares (2), reingresos por insuficiencia cardiaca (2), tras implante de desfibrilador automático implantable (DAI) (1). El resto no dieron explicaciones, no respondiendo a nuestros intentos de comunicación telefónica y/o por correo.

\section{Año 2020}

La duración media de los programas fue significativamente mayor que en el 2019. La totalidad de los pacientes estaban muy afectados por el miedo a contagiarse de COVID-19. La alta incidencia de faltas de 
asistencia, relacionadas directamente con la evolución de la pandemia (contagio, confinamiento, fases de desescalada, etc.) y el miedo a desplazarse en transporte público fueron las principales responsables.

Es de interés tomar en cuenta que las anomalías en la asistencia al gimnasio podrían ser consecuencia de que los pacientes fueron sometidos a diferentes PRC durante el año 2020 (Fig. 2).

Estas modificaciones en los PRC se sumaron a las previamente indicadas. Cumplir con el número de sesiones de entrenamiento programadas (24-36), en 2-3 meses, como se hace de forma habitual, no ha sido posible, por lo que ha sido necesario prolongar la duración.

Abandonaron 15 enfermos: cinco después de la entrevista inicial; tres entre la cuarta y octava semana, uno de ellos se infectó por el SARS-CoV-2, asintomático, y no se reintegró; seis en los primeros 15 días, sin explicar las razones; y uno tras reinfartarse al suspender el tratamiento antiagregante por una cirugía urológica.

Catorce pacientes se infectaron con el coronavirus, siete antes de iniciar el programa y siete durante este. Los procesos fueron leves y los pasaron en su casa, a excepción de tres del grupo de infección previa que precisaron ingresar por neumonía. No existió contagio a los compañeros del grupo de ejercicios ni a los profesionales de la unidad.

La capacidad física mejoró significativamente en los dos grupos tras el entrenamiento, como ocurre de forma habitual. Sin embargo, al comparar los valores iniciales y finales, en el 2020 la puntuación media de los MET fue inferior. No existieron diferencias en los otros parámetros (positividad clínica y/o eléctrica, incidencias de arritmias y respuesta tensional) analizados con las ergometrías, inicial y final (Tabla 2).

Llama la atención que los niveles de ansiedad y las puntuaciones de Bortner fueron significativamente más altas en el año 2019, al comparar los valores al inicio y fin del programa. Los niveles de depresión no mostraron diferencias (Tabla 3). Cinco pacientes en el año 2019 y dos en el 2020 precisaron de tratamiento especializado.

La cardiopatía isquémica fue la patología más frecuente, como ocurre habitualmente, en nuestra URC. Incluye a pacientes con síndrome coronario agudo con elevación del ST (SCACEST), síndrome coronario agudo sin elevación del ST (SCASEST) y con clínica de angina de esfuerzo. Todos ellos fueron estudiados hemodinámicamente y revascularizados de forma percutánea o quirúrgica (Tabla 4).



Figura 2. Número de pacientes incluidos en el Programa de Rehabilitación Cardiaca durante el año 2020, en distintas fases, y dependientes de la evolución de la pandemia de COVID-19. COVID-19: enfermedad por coronavirus 2019; PRMC: programa de rehabilitación multifactorial clásico; TRH: telerrehabilitación; PRA: programa de reapertura.

En el SCA se analizó el tiempo transcurrido entre el inicio del episodio isquémico y la revascularización (código infarto). En el año 2019, 26 de los 50 pacientes (52\%) con SCA se trataron antes de las 8 horas, en el 2020 lo fueron 17 de 67 (25.3\%), diferencia no significativa, por el corto número de casos.

La cirugía valvular se realizó en cardiopatías reumáticas (seis en el 2019 y cuatro en el 2020), y en lesiones aórticas degenerativas (seis en 2019 y tres en el 2020). En las valvulopatías reumáticas se implantaron seis prótesis mitrales y dos aórticas (año 2019) y cuatro mitrales y una aórtica en el 2020. En la cirugía de aortas degenerativas se implantó tubo aórtico en tres pacientes, dos y uno respectivamente.

El apartado de otros diagnósticos incluye a pacientes con una o varias de estas anomalías: presencia de múltiples factores de riesgo y patología ateroesclerosa periférica, obesos con capacidad funcional muy reducida, ablación de fibrilación o flutter auricular, portadores de marcapasos o DAI.

\section{Discusión}

Muchas de las URC en hospitales públicos y privados han tenido que cerrar, por las razones previamente descritas. Las unidades extrahospitalarias, como la nuestra, han podido permanecer abiertas porque sus instalaciones y su personal no pueden asumir el tratamiento de los pacientes infectados.

Las características generales de los pacientes incluidos en nuestra unidad son similares en ambos años 
Tabla 2. Características prueba de esfuerzo

\begin{tabular}{|l|c|c|c|c|c|c|c|c|}
\hline Variable & \multicolumn{3}{|c|}{ Año 2019} & \multicolumn{3}{c|}{ Año 2020 } & \multicolumn{2}{c|}{ p 2019 vs. 2020} \\
\hline & Inicio & Alta & P & Inicio & Alta & p & Inicio & Alta \\
\hline MET, media (DE) & $8.3(2.1)$ & $10(2.5)$ & 0.001 & $7.8(2)$ & $9.5(2.2)$ & 0.001 & NS & 0.001 \\
\hline
\end{tabular}

*Las variables se expresan en media (desviación estándar). Diferencias estadísticamente significativas.

$P<0.05$. No existieron diferencias significativas en el resto de los parámetros (positividad clínica y/o eléctrica, arritmias y respuesta tensional).

MET: unidad de medida del índice metabólico (cantidad de calor emitido por una persona en posición sedente por metro cuadrado de piel); DE: desviación estándar;

NS: no significativa.

Tabla 3. Características de los pacientes con alteraciones psicológicas

\begin{tabular}{|l|c|c|c|c|c|c|}
\hline Variable & \multicolumn{2}{|c|}{ Año 2019} & \multicolumn{2}{c|}{ Año 2020 } & \multicolumn{2}{c|}{ p } \\
\hline & Inicio & Alta & Inicio & Alta & Inicio & Alta \\
\hline Ansiedad, $n(\%)$ & $55(46.6)$ & $33(31.1)$ & $35(32.4)$ & $13(12.8)$ & 0.024 & 0.002 \\
\hline Depresión, $n(\%)$ & $24(20.3)$ & $13(12.2)$ & $22(18.8)$ & $8(7.9)$ & NS & NS \\
\hline PCTA, media (DE) & $152.5(48.1)$ & $144.7(55.4)$ & $137.6(54.7)$ & $126.5(54.7)$ & 0.041 & 0.034 \\
\hline
\end{tabular}

*Las variables cualitativas se expresan en número (porcentaje), las variables cuantitativas en media (desviación estándar). Diferencias estadísticamente significativas. $\mathrm{P}<0.05$.

DE: desviación estándar; n: número; NS: no significativa; PCTA: patrón de conducta tipo A.

Tabla 4. Características de los pacientes con cardiopatía isquémica

\begin{tabular}{|c|c|c|c|}
\hline Variable & $\begin{array}{c}\text { Año } 2019 \\
\text { (99 pacientes) }\end{array}$ & $\begin{array}{c}\text { Año } 2020 \\
\text { (106 pacientes) }\end{array}$ & $\mathbf{p}$ \\
\hline FEVI, media (DE) & $55.8(10.8)$ & $55.9(10.1)$ & \multirow{8}{*}{ NS } \\
\hline RPC completa, $n(\%)$ & $70(70.7)$ & $73(68.8)$ & \\
\hline RPC incompleta, $\mathrm{n}(\%)$ & $22(22.2)$ & $25(23.5)$ & \\
\hline $\mathrm{R} 0$ completa, $\mathrm{n}(\%)$ & $5(5)$ & $7(6.6)$ & \\
\hline $\mathrm{R} 0$ incompleta, $\mathrm{n}(\%)$ & $2(2)$ & $1(0.9)$ & \\
\hline Tiempo hasta revascularización, media (DE) & $14.3(28.2)$ & $10.3(17.7)$ & \\
\hline $1-4 h, n(\%)$ & $24(24.2)$ & $15(14.1)$ & \\
\hline $5-8 \mathrm{~h}, \mathrm{n}(\%)$ & $2(2)$ & $2(1.8)$ & \\
\hline
\end{tabular}

*Las variables cualitativas se expresan en número (porcentaje), las variables cuantitativas en media (desviación estándar). Diferencias estadísticamente significativas. $\mathrm{P}<0.05$. percutánea.

analizados. La duración media del PRC en el 2020 ha aumentado, llegando a ser de 4-6 meses en un alto porcentaje de casos. Es consecuencia de la evolución de la enfermedad, de los confinamientos y del miedo al contagio por parte de los pacientes. Muchos de ellos han desaparecido durante semanas, para reaparecer posteriormente y completar el programa. Cuatro no iniciaron el tratamiento al tener que desplazarse en transporte público.

El número de abandonos del programa fue superior en los pacientes tratados en el año 2020. Los pacientes admitieron que la razón fue el miedo al contagio de la enfermedad. En los del año 2019 la causa fundamental fue la falta de motivación. 
Otra faceta que considerar es la que se refiere a los pacientes que sufrieron la COVID-19, antes o durante el PRC. El tiempo medio de llegada a la unidad fue superior a los tres meses, en los previos. Los contagiados durante el programa se remitieron a sus médicos de familia tras las llamadas telefónicas de los pacientes o inmediatamente (5-10 minutos) después de que llegaran al gimnasio con síntomas compatibles con un posible contagio. Tras la infección los pacientes regresaron a las 2-4 semanas de una PCR negativa, en función de la severidad de los síntomas.

Un elevado porcentaje de las personas que han padecido COVID-19 refieren, tras la recuperación de la fase aguda de la enfermedad, manifestaciones clínicas tanto subjetivas como objetivas que se prolongan más allá de las tres semanas, llegando a persistir durante meses. Se han sugerido distintos términos a la persistencia de esta sintomatología (astenia, disnea, tos, molestias musculares torácicas y de índole general) como COVID postaguda, prolongada o crónico, aunque el más admitido es el de síndrome post-COVID (SPC) ${ }^{11}$. Es relativamente frecuente en aquellos adultos que han precisado ingreso en unidades de cuidados intensivos.

Carfi, et al. ${ }^{12}$ analizan la clínica en el periodo tras la hospitalización de 143 pacientes ingresados por COVID-19. A los 60 días del inicio de la enfermedad, solo el $13 \%$ estaba asintomático. Las manifestaciones respiratorias eran las más frecuentes. El metaanálisis de Torres-Castro, et al. ${ }^{13}$ revisa siete estudios que incluyen a 380 pacientes con COVID-19, encontró la presencia de un patrón respiratorio restrictivo hasta en un $20 \%$ de los casos y alteración de la capacidad de difusión hasta en un $40 \%$.

En nuestro estudio, la capacidad física medida en las pruebas de esfuerzo inicial y final, con similar fracción de eyección, es más baja en el año 2020. No puede descartarse la existencia de SPC en los 14 pacientes que habían presentado COVID.

La irregularidad en la asistencia al entrenamiento presencial durante la época COVID-19 pudo ser también otra de las causas responsables, pese a que el porcentaje de pacientes que hacían el programa de marchas domiciliario fue superior en el año 2020 (89\%) al compararlo con el 2019 (78\%), con una $p=0.017$.

Creemos que la mascarilla puede jugar un papel primordial en la menor capacidad física. Más del $90 \%$ de los pacientes se quejaron de su uso durante el entrenamiento y en la prueba de esfuerzo.

Se han descrito trastornos psicológico-psiquiátricos de concentración 0 atención, de memoria, labilidad emocional, ansiedad, irritabilidad, fatiga y síntomas relacionados con estrés postraumático en un análisis de más de 62,000 afectados por COVID-19 ${ }^{14}$.

Los niveles de ansiedad y las puntuaciones del patrón de conducta tipo $A$, en nuestros pacientes, fueron superiores en el año 2019. Los trastornos psicológicos (ansiedad y depresión) son muy habituales en las primeras semanas que siguen a un SCA. Se caracterizan por molestias torácicas, que no recuerdan a los del episodio agudo y se acompañan de sensación de falta de aire e imposibilidad de suspirar profundamente. Suelen desaparecer después de realizar la ergometría y tras dos o tres semanas de entrenamiento.

Creemos que la razón para las diferencias entre los dos años fue que llegaron a realizar el programa de forma más tardía, por dificultades en las revisiones cardiológicas y en la decisión de indicar la RC por parte de los cardiólogos. Podría decirse que la frecuente ansiedad postinfarto ha pasado a ser miedo por el contagio. El miedo a la COVID es el responsable de que las puntuaciones del Bortner sean inferiores en el año 2020, entendible si analizamos las distintas cuestiones que se preguntan. Las ambiciones se reducen al predominar la sensación de no tener el futuro asegurado.

Según distintos consensos, es necesario estratificar el riesgo de los pacientes antes de recomendar la reanudación de la actividad física especialmente si persisten síntomas ${ }^{15}$.

Diversas sociedades de la Federación Europea de Medicina Deportiva, en diferentes publicaciones, describen que: a) antes de reiniciar el deporte para deportistas, las actividades de la vida diaria deben ser fácilmente alcanzables y la persona debe ser capaz de caminar $500 \mathrm{~m}$ sobre el piso sin sentir excesiva fatiga o disnea; b) el regreso al ejercicio o la actividad deportiva puede realizarse después de un periodo asintomático de al menos siete días, pero es necesario un análisis más profundo en las actividades físicas intensas, y c) debe restringirse el ejercicio durante tres a seis meses en casos de miocarditis confirmada por resonancia magnética cardiaca o biopsia miocárdica ${ }^{16-18}$.

Con respecto a los síntomas respiratorios, la tos persistente y la disnea, suele desaparecer después de varias semanas. Los síntomas progresivos que no se resuelven o que empeoran deben ser estudiados en profundidad.

La terapia ocupacional debe ocupar un importante lugar en la rehabilitación de los pacientes post-COVID, ya que facilitan la reincorporación sociolaboral de los enfermos. 
La utilización de la escala de Börg es de suma importancia en el entrenamiento de los enfermos que han estado contagiados por la COVID. La sensación subjetiva de esfuerzo complementa a la objetiva medida con la FCE.

El entrenamiento aeróbico debe efectuarse de forma progresiva, en tiempo e intensidad. El de fuerza podría iniciarse después de 3-4 semanas, en ausencia de síntomas.

\section{Conclusiones}

Creemos que el presente estudio demuestra que es posible continuar realizando los PRC en época COVID-19, aunque sea necesario modificar y adaptar las actuaciones, con base en la evolución epidemiológica y política de cada momento. Los resultados obtenidos no difieren de forma significativa de los PRC efectuados en los años previos. Aunque la situación actual afecta de forma preferente a los programas de la medicina pública, nos satisface saber que las URC seguirán abiertas, dado el desarrollo de este tipo de tratamiento en los últimos años en nuestro país.

\section{Financiamiento}

El presente trabajo no ha recibido ninguna beca específica de agencias de los sectores público, comercial o sin ánimo de lucro.

\section{Conflicto de intereses}

Los autores declaran que no tienen conflicto de intereses.

\section{Responsabilidades éticas}

Protección de personas y animales. Los autores declaran que para esta investigación no se han realizado experimentos en seres humanos ni en animales.

Confidencialidad de los datos. Los autores declaran que han seguido los protocolos de su centro de trabajo sobre la publicación de datos de pacientes.
Derecho a la privacidad y consentimiento informado. Los autores han obtenido el consentimiento informado de los pacientes y/o sujetos referidos en el artículo. Este documento obra en poder del autor de correspondencia.

\section{Bibliografía}

1. Rodriguez-Leor $O$, Cid-Alvarez AB, Perez del Prado A, Rosello $X$, Ojeda E, Serrador A, et al. In-hospital outcomes of patients with ST-segment elevation myocardial infarction and COVID-19. Eurolntervention. 2021;16(17):1426-33.

2. Rodriguez-Leor O. Infarto y COVID-19. La estrecha relación entre el infarto y la COVID-19: la mortalidad durante el ingreso hospitalario por infarto se dobló en la primera ola de pandemia. Ciencia Cardiovascular. 2021;140:36-42

3. Marcadet D, Bruno $P$, Richard L. Réadaptation cardiaque en période épidémique de COVID-19. Propositions du GERS-P le 06/05/2020 [Internet]. Société Française de Cardiologie. Disponible en: sfcardio.fr/sites/ default/files/2020-05/2020-05-06-Réadaptation_cardiaque

4. Recomendaciones de la Asociación de Riesgo Vascular y Rehabilitación Cardiaca de la Sociedad Española de Cardiología (SEC) y Sociedad Española de Rehabilitación Cardiorrespiratoria (SORECAR) para reiniciar las actividades de Rehabilitación Cardiaca en la situación de desescalada por COVID-19 en España [internet]. Sociedad Española de Cardiología, Asociación de Riesgo Vascular y Rehabilitación Cardiaca; 8 de mayo de 2020. Disponible en: svmefr.com/wp-content/uploads/2020/05/ recomendaciones-rehabilitacion-cardiaca-SEC-2020-COVID19.pdf

5. Hamilton $\mathrm{M}$. The assesment of anxiety states by rating scales. $\mathrm{Br} \mathrm{J}$ Med Psychol. 1959;32:50-5

6. Beck AT, Ward $\mathrm{CH}$, Mendelson M, MockJ, Erbaugh J. An inventory for measuring depression. Arch Gen Psychiatry. 1961;4:561-71.

7. Bortner RW. A short rating scale as a potential measure of pattern "A" behaviour. J Chronic Dis. 1969;22:87-91.

8. Alonso A Carcedo C. Pautas de estudio y tratamiento psicológico. En: Maroto Montero JM, de Pablo Zarzosa C, editores. Rehabilitación Cardiovascular. Madrid, España: Editorial Médica Panamericana; 2010. pp. 273-290.

9. Borg G. Perceived exertion as an indicator of somatic stress. Scand J Rehabil Med. 1970;3:82-8.

10. Maroto JM. Indicaciones y protocolos actuales de rehabilitación cardiaca. En: Maroto JM, editor. Rehabilitación cardiaca. Madrid, España: Sociedad Española de Cardiología; 2009. pp. 1-17.

11. Bouza E, Cantón R, Ruiz J, Rodriguez F, Gómez J, García A, et al. Síndrome Post-Covid del Colegio de Médicos de Madrid. Un documento para la reflexión [Internet]. Comité Científico del Ilustre Colegio Oficial de Médicos de Madrid. Disponible en: https://www.icomem.es/adjuntos/ adjunto_3021.1615816967.pdf

12. Carfi A, Bernabei R, Landi L; Gemelli Against COVID-19 Post-Acute Care Study Group. Persistent symptoms in patients after acute COVID-19. JAMA. 2020;324:603-5.

13. Torres-Castro R, Vasconcello-Castillo L, Alsina-Restoy $X$ Solis-Navarro L, Burgos F, Puppo H, et al. Respiratory function in patients post-infection by COVID-19: a systematic review and meta-analysis. Pulmonology. 2021;27(4):328-37.

14. Taquet M, Luciano S, Geddes JR, Harrison PJ. Bidirectional associations between COVID-19 and psychiatric disorder: retrospective cohort studies of 62354 COVID-19 cases in the USA. Lancet Psychiatry. 2021;8(2):130-40.

15. Salman D, Vishnubala D, Le Feuvre P, Beaney T, Korgaonkar J Mageed A, et al. Returning to physical activty after COVID-19. BMJ. 2021 Jan 8;372:m4721.

16. Löllgen H, Bachl N, Papadopoulou T, Shafik A, Holloway G, Vonbank K, et al. Recommendations for return to sport during the SARS-CoV-2 pandemic. BMJ Open Sport Exerc Med. 2020;6:e000858.

17. Hull JH, Loosemore M, Schwellnus M. Respiratory health in athletes: facing the COVID-19 challenge. Lancet Respir Med. 2020;8:557-8.

18. Elliott N, Martin R, Heron N, Elliott J, Grimstead D, Biswas A. Infographic. Graduated return to play guidance following COVID-19 infection. $\mathrm{Br} J$ Sports Med. 2020;54:1174-5 